\title{
Quantum Coherent Entangled Hydrogen Atom Non-Locality
}

\author{
Mark Moody \\ Independent Researcher, Denver, USA \\ Email: wavepatternmath@gmail.com
}

How to cite this paper: Moody, M. (2020) Quantum Coherent Entangled Hydrogen Atom Non-Locality. Journal of Applied Mathematics and Physics, 8, 737-752. https://doi.org/10.4236/jamp.2020.85057

Received: February 3, 2020

Accepted: April 23, 2020

Published: April 26, 2020

Copyright (c) 2020 by author(s) and Scientific Research Publishing Inc. This work is licensed under the Creative Commons Attribution International License (CC BY 4.0).

http://creativecommons.org/licenses/by/4.0/

(c) (i) Open Access

\begin{abstract}
The discovery of a data based informational wave pattern infuses coherent entanglement into the system. This discovery of how to add coherent entanglement into the model provides the missing key, that has opened the door to understanding the universe. It is found that the inclusion of coherence and entanglement at the start of the system is extremely simple and in fact it is so simple that it has simply been overlooked. Entanglement and coherence are the most fundamental aspects of our universe. It is demonstrated that the basic model of the hydrogen atom is made from the CMB. If we add entanglement into this basic model of the hydrogen atom a math system called Wave Pattern Entangled Math is unveiled. This system of wave interference mathematics creates a data system in which entanglement and coherence can easily be understood. The final outcome is an unbreakable pattern of information, including entangled energy, entropy, spin, universal expansion, compression, velocity of light, C2, and Quantum Coherence.
\end{abstract}

\section{Keywords}

Entropy Function, Entanglement, Entangled, Universal Matrix, Wave Pattern Entangled Math, WPEM, Complementarity, Causality, Wave-Particle Duality, Hydrogen Atom, C.M.B., Cosmic Microwave Background Wave, Frequency

\section{Introduction}

The standard model of the hydrogen atom was discovered and agreed upon by many of the most prominent figures in science.

Scientists reached a point that stopped them. This failure point is because they all failed to discover the entanglement and the coherent pattern in their model of the hydrogen atom. If the former scientists of the 40s and 50s had a better understanding of entanglement and coherence they would have been able to con- 
tinue this simple explanation of reality showing the $\mathrm{CMB}$ makes the hydrogen atom that expands to the make the entire universe in a single thermal dynamic interior system.

This failure point is why the science turned to particle, imaginary numbers, invented math systems and explanations of reality which are so complicated that no one can understand them. Yet, not one of these explanations has told us the most fundamental aspects of the universe. Such as, what the universe is.

\section{It Is Very Clear What the Universe Is}

The most basic building block of the universe is the hydrogen atom. $99.9 \%$ of everything in our universe is hydrogen atoms.

99.9\% of an apple is apple. An apple is not made from cherries.

The universe is made of hydrogen! Thus, it must be a hydrogen atom!

This is what all the great scientists were trying to prove. However, at the time they were proving the universe is a hydrogen atom they did not know about entanglement or coherence. If the scientist in the 40 s and 50 s had a better understanding of entanglement they would have certainly finished the universal hydrogen atom model, and we could have avoided the confusion of the last 50+ years.

The crux of this argument rests on the fact that the universe not only abides by the thermal dynamic laws but creates them.

This means no energy, force, momentum, spin or anything is ever added or removed from the system. The system and all elements are expanded and compressed in a continuum that does not allow any element to act independently in the system. A change in one will change all other elements.

In this type of system everything that is in the system (energy, charge, momentum, spin, force, etc.) must be present and fully formed at the start of the system. For instance, we know the system has force, therefore that force must be present at the very start, and we can expand or stretch, compact, transpose, mirror this force, but we can never add or take away force.

This is true of data, information, entanglement and coherence. These things must be present at the start of the system, and they must be fully formed.

That is to say all the information or data needed for the universe to expand has to be there at the start. This makes sense if the universe is hydrogen atom all the information is exactly the same in a tiny hydrogen atom or universe size hydrogen atom. We also know that the energy in one tiny hydrogen atom is the same as the energy of the entire universe. (The Hydrogen Bomb released only a tiny amount of energy in the hydrogen atoms.)

In this paper we will demonstrate that all things needed to make the universe are in the hydrogen atom.

We need not invent any math to prove this argument. When we simply follow the work of the former great scientist and add in the modern elements such as entanglement and coherence. 


\section{Spectra of a Hydrogen Atom}

Heisenberg showed that the atomic Spectra of a hydrogen atom is based on the numbers $1,2,3,4,5,6,7,8,9$. Ritz noticed that they were fundamental frequencies. These fundamental frequencies were further found to fall into groups, the numbers in any one of those groups being associated with the series of integers $1,2,3,4,5,6,7,8,9$ [1] [2].

When the single electron of a hydrogen atom is in its innermost orbit it is said to be in the normal state, $\mathrm{n}=1$ the next orbit is $\mathrm{n}=2,3,4,5,6,7,8,9$.

Schrödinger, Heisenberg, and Dirac all came up with methods to calculate the frequencies of the hydrogen atom and in the end, they all came up with the shells of the hydrogen atom in the perfect pattern of $1,2,3,4,5,6,7,8,9$ [2] [3].

The below is a harmonic series wave that is created from a microwave such as found in the cosmic microwave radiation background wave. A microwave is the only wave that has a wavelength of $1 \mathrm{~mm}$ [4]. Exactly what is needed to make the wave shown in Figure 1. In order for our system to work, we must start with a perfect harmonic series wave.

The integers of $1,2,3,4,5,6,7,8,9$ are a harmonic series. Harmonic series. $\mathrm{Hn}=1 / 2,2 / 3,3 / 4,4 / 5,5 / 6,6 / 7,7 / 8,8 / 9, \ldots$

\section{Entanglement}

When two particles are entangled they displayed mirrored characteristics, like Spin and charge even when separated by billions of light-years. This makes them complementary. A change in one particle is instantly mirrored in the other [5].

We simply mirror the pattern as shown in Figure $1-0123456789$ to 9876543210. The pattern becomes 0123456789876543210 .

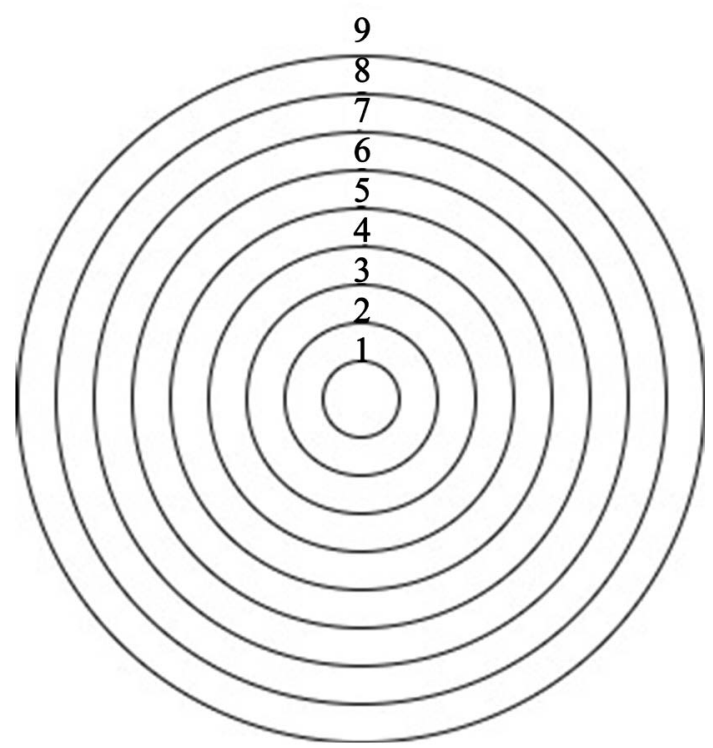

Figure 1. This is the harmonic wave that must be made from a microwave. No other wave dispersion is possible in space as long as no exterior forces are acting upon this wave. The C.M.B. is a Microwave. 
This simple method has entangled the pattern of the hydrogen atom we can easily find the hydrogen spectrum.

The pattern 0.012345678987654321 inverted $=1 / 012345678987654321=$ $8.1000000162000000243000000324 \mathrm{e}-17 \ldots$

This is now the spectrum pattern for the hydrogen atom. With one simple division, we have found the harmonic series entangled frequencies of the hydrogen atom.

Notice $81,162,243,324,-6561 \ldots$ are harmonic series still we have not broken the pattern.

This pattern starts with 81 being harmonic series it must extend to 81 squared $=6561$.

This 6561 is important because it is the exact measured spectral line from a hydrogen atom.

Bohr, Schrödinger, Heisenberg, and Dirac all came up with methods to calculate the frequencies of the hydrogen atom and in the end, they all came up with almost the exact same measurements as Bohr shown below.

656.1 first Hydrogen spectrum line [1].

The other scientist Measure the first line of the hydrogen spectrum to be 656.1

$$
\sqrt{6561}=81
$$

Equation (1)

With Wave pattern entanglement math as in Equation (1), it is found that $8.1000000162000000243000000324-6561 \ldots=$ Harmonic series entangled hydrogen spectrum $81^{2}=6561$. The measured amount is in complete agreement with our simple inverse spectrum solution.

At 81 , the system is at a smaller dimension than the larger hydrogen atom at 6561 that they measured but the pattern is still exactly the same.

This is the very point that causality physics drops off. All the same, minds arriving at the same conclusion but without entanglement there system or pattern broke.

Not making the simple inclusion of entanglement into the hydrogen atom structure led to the inability to continue the pattern and led to more complicated non-solutions such as Quantum Mechanics, Particle physics and a thousand other ways to explain the break in the pattern. Simply because, we did not add entanglement into the pattern.

With entanglement, our pattern continues to flow, whereas the static model of the atom with its shells end. They can go no further with it. Using harmonic series wave pattern entanglement math, I can continue to build the hydrogen atom spectrum.

By simply using the entangled pattern we don't actually need math we can just treat the shell numbers, as waves and combine the waves.

$\mathrm{U}=$ Union

In Figure 2, you find that every column is harmonic series.

In Figure 2, we also find that the pattern is still entangled 09183645546372 8190 is mirrored 09-90, 18-81, 36-63, 45-54. This is entangled, and the pattern 


$\begin{array}{ll}\{0\} \mathrm{U}\{9\}=\{09\} & \{09\} \mathrm{U}\{18\}=\{1089\} \\ \{1\} \mathrm{U}\{8\}=\{18\} & \{18\} \mathrm{U}\{27\}=\{2178\} \\ \{2\} \mathrm{U}\{7\}=\{27\} & \{27\} \mathrm{U}\{36\}=\{3267\} \\ \{3\} \mathrm{U}\{6\}=\{36\} & \{36\} \mathrm{U}\{45\}=\{4356\} \\ \{4\} \mathrm{U}\{5\}=\{45\} & \{45\} \mathrm{U}\{54\}=\{5445\} \\ \{5\} \mathrm{U}\{4\}=\{54\} & \{54\} \mathrm{U}\{63\}=\{6534\} \\ \{6\} \mathrm{U}\{3\}=\{63\} & \{63\} \mathrm{U}\{72\}=\{7623 \\ \{7\} \mathrm{U}\{2\}=\{72\} & \{72\} \mathrm{U}\{81\}=\{8712\} \\ \{8\} \mathrm{U}\{1\}=\{81\} & \{81\} \mathrm{U}\{90\}=\{9801\}\end{array}$

Figure 2. We see the entanglement and the perfect harmonic series pattern is continued. No math is needed it just a continuation of the pattern.

has not been broken it is still in perfect Harmonic series. In the last column again we find the pattern remains entangled as it is mirrored 1089-9801, $2178-8712,3267-7623,4356-6534$ until we reach the center of 5445 this center exactly mirrors itself as $54-45$.

\section{It Is Possible to Make a Visual Representation of This Hydrogen Atom Spectrum}

The graph in Figure 3 is made from the calculus form of the Zeta function.

It is a way of combining the Fourier wave transformation approach to the problem of demonstrating infinite solutions in a finite system. The Zeta Function I use is expressed as a sum of sine and cosine waves at different frequencies as sine and cosine are basically harmonic functions. What it does is simply convert the Matrix into frequency domains. It also adds a function of time into the function of frequency. This allows for a force to be calculated that takes the spin into account. This conversion of spatial coordinates do to the special frequency domain provides a spectacular visual representation of the holographic Universe via the animated polar graph.

Following each Dynamic Point as it moves due to the dynamic nature of the system. The graph shows that this point is moving in a straight line.

You can see the animated graph and understand it much better by watching the video on YouTube at this address: https://youtu.be/rg4tb4ibRYs.

\section{Stages of Figure 3}

STAGE 1: Light frequency or rays are passed to the center all arriving at a point less than 1 .

STAGE 2: The rays are manipulated by the forces into the center of the system and reflected back out into a two-dimensional lotus flower.

STAGE 3: The magnetic lines are actually created in what is known as a Langmuir sheath. This type of magnetic field is found in nature from solar flares to the Earth's magnetic field, it's literally everywhere.

STAGE 4: We see that spin has now been added to the system and the spiral galaxy pattern appears just as one would need and expect in an accurate model of the universe. 


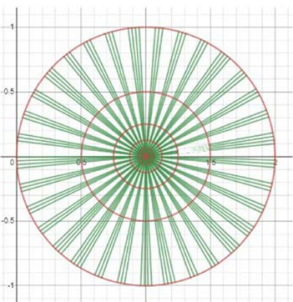

Stage 1

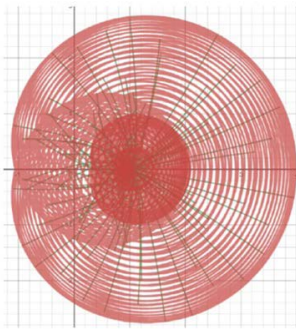

Stage 3

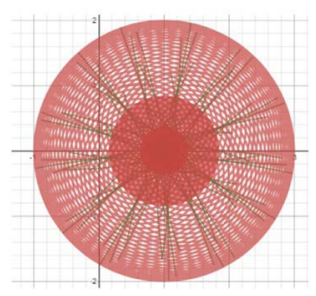

Stage 2

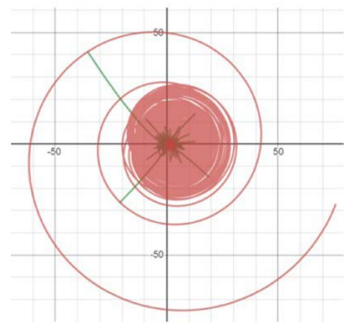

Stage 4

Figure 3. This hydrogen atom spectrum does perfectly create a system that mirrors our universe and galaxies. Keep in mind this a continuous animated graph nothing was done other than input the matrix from Figure 2 into the zeta equation and hit start. The fact that it makes exactly what we would expect to find as a model for universe cannot be coincidence!

This is an internal closed system that was able to build what appears to be a model of the universe without ever adding anything to system as it expanded.

This demonstrates what must come from a harmonic series entangled wave in space nothing else is possible and it is impossible for it not to create a system similar to what I have shown here.

That is quite an amazing result, however, it's not done. In this same animated graph made from one single Matrix, we can enter into the compression zone or the black hole and discover what is happening to the pattern and eventually arrive at the loom or the Creator of this entire system.

By adding in the entanglement I will show that we can simply reduce all the participants in the system not just particles but light, force, energy, spin, acceleration into a single point wave-particle that and graphically display this point.

\section{LOGOS Nucleus of the Hydrogen Atom-Point Particle}

Riemann Zeta Function mapped from $\mathrm{z}=\mathrm{a}+\mathrm{bi}, .0001<\mathrm{a}<81, .1008<\mathrm{b}<$ 1008. The distorted grid lines represent where a grid defined on $\mathrm{z}$ would map to after applying the zeta function in Figure 4.

The red circles in Figure 4 are the entangled hydrogen spectrum starting with $\mathrm{a}=012345678987654321$

09182736455463728190

108921783267435654456534762387129801

The green lines in Figure 4 are the single frequency of light a photon at the exact measured photon energy level of a microwave at 121 entering into the sys- 
tem.

I used the hydrogen spectrum 9, $1827,36,45,54,63,72,81,90$ in and the entangled photon energy constant 121 in b to make Figure 4.

As we enter into the center mass of Figure 3 stage 4 and begin to reduce. A mathematical entity or the loom, can clearly be seen spinning these frequencies into a web that expands into the universe.

This entity is no more than the size of a proton but contains incredible energy and forces that are being exerted upon it from the continuum. We find that this entity is based on a harmonic wave as it appears to have nine arms, 9 legs, nine teeth.

$1 / .111111111=9$

Reminder; all these stages of Figure 3 and Figure 4 came from one simple harmonic entangled microwave wave.

From the simplest thing in the universe comes the complexity to create our entire reality.

By graphing the entangled harmonic series wave of the hydrogen atom I have taken complicated system of elements and reduced them to a single point particle with the total mass located in the center of the system.

By moving the external force to the center we now have a closed thermal dynamic system.

This is what happens to a harmonic series entangled wave in space when you reduce it to a point particle.

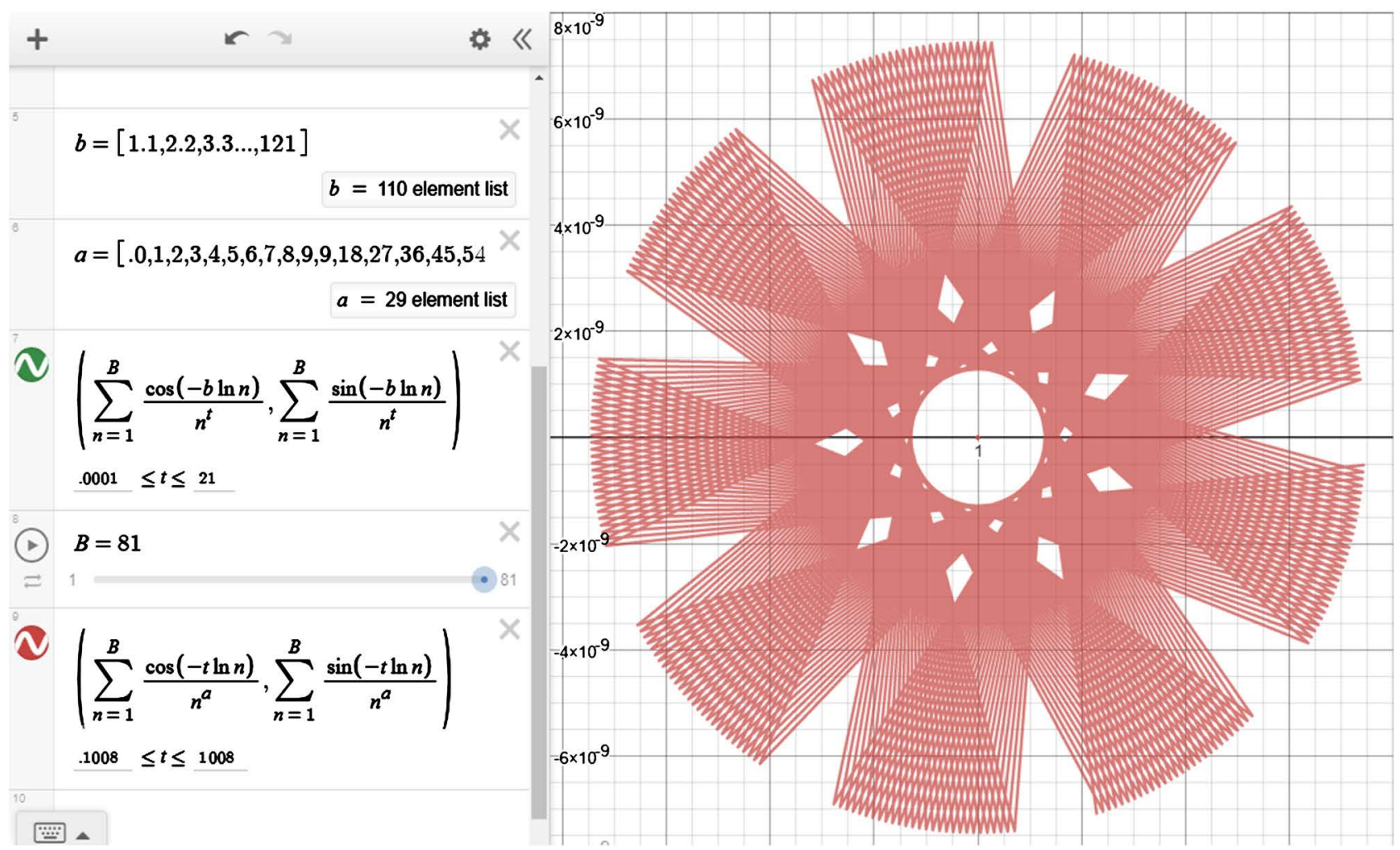

Figure 4. This entity is what is actually found as we reduce the graph down 11 trillion times smaller than Stage 4. 
The key element here is that entanglement was introduced into the system at the start. Thus, it is able to continue in the system as entanglement is what created the system. It is still the hydrogen atom!

With new insight into how simply information can be added to the system the following functions can be derived.

\section{Entangled Action and Reaction}

Newton's third law tells us that the action must be equal to the reaction but it does not say it can't be divided up into different parts. The action and reaction can and must be divided up into a minimum of three parts to create a three-dimensional reality. The sum of those parts will equal the total action or at least mirror exactly the total action.

Newton's third law is two-dimensional, it describes a flat wave that will expand and compress its center in two-dimensional space and meet his requirements.

We live in three-dimensional space so there must be another dimension in the action and reaction and there is the space between the two poles is now charged and that space has energy, force, velocity, mass, spin and now that space between the points is pure potential having everything needed to create anything we can imagine.

Now finally we can calculate this entangled space because we know that everything between these two poles has to be entangled. The energy has to be propagating at the same rate as the force! Spin, velocity mass all must be propagating completely dependent upon the other elements in this continuum.

We can now know that this difference between the action and reaction has a numerical value and can be obtained as demonstrated in Equation (2).

$$
\frac{\frac{\text { action }}{1}}{\text { action }}=\text { entangled reaction }
$$

Equation (2)

\section{Complementarity}

Equation (2) demonstrates that Complementarity is more fundamental than relativity, which takes the speed of light as an absolute limit. This means that entanglement is more fundamental than the four basic forces in nature [6].

The coexistence of opposites is called complementary. In any situation in which opposites exist, one can replace the other in specific circumstances, and at the same time maintain aspects such as negative and positive charge, spin, energy, and momentum that are completely dependent upon the other. Any change in one will result in a change of all participants in this entangled continuum.

Einstein's theory of relativity 1905 is a deduction from the observed pattern of events.

It is causality. 
Complementarity: (When two particles are entangled they displayed mirrored characteristics, like Spin and charge even when separated by billions of light-years. This makes them complementary. A change in one particle is instantly mirrored in the other).

Complementarity being more fundamental than relativity (Causality)shows us the nature of these relationships and that it is a wave and that this wave is entangled.

Einstein was unable to include entanglement in his equation, and he had to borrow the Complementarity entangled constant of $c^{2}$ in order to make his relativity work. Relativity only works because it actually borrows $E=m c^{2}$ from Complementarity.

$E=m c^{2}$ does not prove relativity. It actually disproves relativity and proves Complementarity. The $c^{2}$ is not possible in relativity $\mathrm{c}$ is the limiting factor in relativity, only in a Complementarity system do we find $c^{2}$. Only by borrowing the $c^{2}$ from Complementarity can he make relativity work.

The general "principle of locality" requires that "for an action at one point to have an influence at another point, something in the space between the points, such as a field, must mediate the action". In view of the Theory of Relativity, the speed at which such an action, interaction, or influence can be transmitted between distant points in space cannot exceed the speed of light. This formulation is also known as "Einstein locality" or "local relativistic causality". It is often stated as "nothing can propagate faster than light, be it energy or merely information" or simply "no spooky action-at-a-distance", as Einstein himself put it [6].

Now it is possible to define $C$. The speed of light squared has been used in $e=$ $m c^{2}$ to calculate the most important and fundamental elements of our reality and is the backbone of relativity. However, $e=m c^{2}$ is not actually a part of relativity but is the backbone of complementarity.

\section{Entangled Velocity of Light}

Equation (3)

$$
\frac{c}{\frac{1}{c}}=c^{2}
$$

Replacing $c$ with the actual numbers for light in Equation (3) we get the below. 300,000/divided by its inverse.

$$
.000003333333333333333333333333333=90,000,000\left(c^{2}\right) \text {. }
$$

Thus, we can say. Equation 3 yields:

$c^{2}$ represents the total entangled velocity of the universe.

$c^{2}$ is real and the velocity constant of Complementarity and not Relativity.

This is possible because the inverse is the opposing pole. The inverse is the reaction to the speed of light. Equation (3) has included entanglement into the speed of light and by doing, so we find that no rules in physics or complemen- 
tarity have been violated, and we now have a universal velocity of light $=c^{2}$.

Light cannot simply be moving in one direction without the opposing reaction. All participants and elements in our universe must abide by the same Laws.

This is extremely important for the first time we have an explanation for the speed of light squared. Instead of just using it because it works. Entanglement demonstrates exactly how $c^{2}$ exist without violating any of the laws of physics.

Furthermore the entangled Velocity of light gives us the hydrogen atom!

Equation (4)

$$
1 \div\left(\frac{c^{2}}{\frac{1}{c^{2}}}\right)=1234567901
$$

The velocity is propagating perfectly in Harmonic Series entangled hydrogen microwave wave as demonstrated by Equation (4).

Thus, if we find the square root of our initial entangled wave Equation (5)

$$
\sqrt{.012345678987654321}=0.111111111
$$

$0.111111111 \times c^{2}=0.012345678987654321$

Inverted $1 / 12345678987654321=81.000000162000000243000000324$

Equation (5) has produced effortlessly the Hydrogen spectrum.

We know that a microwave creates the wave pattern of 0123456789 we know the $\mathrm{CMB}$ is a microwave thus the wave pattern of a microwave when square rooted $=0.11111111$.

This most basic wave 0.111111111 squared $=012345678987654321=$ The entangled CMB wave that makes the hydrogen atom.

This entangled system allows for further simple calculation of entangled energy. We have now discovered one constant, "the universal velocity of light".

\section{Entangled CMB Constant}

We will need to discover at least one more constant and combine them together to see if they do indeed form a continuum and create the energy needed in perfect harmonic series.

We have the velocity of light and the only other thing we can say with any certainty that we have to start the universe with is the CMB.

The CMB is measured at 2.73 degrees kelvin

We are going to simply extend the 2.73 to the more accurate $2.7272727272727272727272727272727=$ Action

This is important when dealing with the quantum world that we don't use exponents. Now we apply

$$
\frac{\frac{\text { action }}{1}}{\text { action }}=\text { entangled reaction }
$$

Equation (2) 
We have now our second constant the entangled thermal temperature of the C.M.B. $=2.7272727272727272727272727272727$

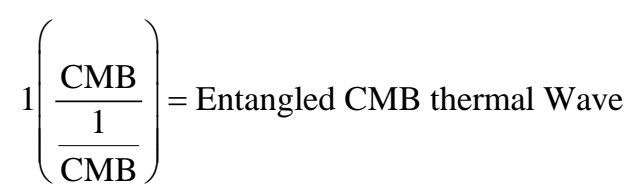

Equation (6)

Entangled CMB thermal wave $=0.134444444444444444444444444444445$ has now been found using Equation (6) and Equation (6) is derived directly from Equation (2).

Now we can combine these two constants to get the entangled universal energy. $0.134444444444444444444444444444445 * \mathrm{C} 2=12,100,000,000$.

That interesting because the energy density of the universe has been measured many times to be $1 \times 10120 \mathrm{~cm}$ cubed. It appears we have a match as $1.008333333 \times 10,120=1,210,000,000$.

This energy also matches the photon energy of a microwave. It needs to match the energy of a microwave and it does perfectly.

These two constants, and the answers are only entangled in a two-dimensional flat wave. It is not until we add in coherence that they become three-dimensional. The coherent factor will be included further down in this paper.

\section{Entangled Entropy Function}

An invariant quality of a closed thermal dynamic system must hold true for all the participants of the system. Any member of the continuum must be present at the very start of the primordial beginnings of the system. Nothing can ever be added. Thus, if we experience entanglement (non-locality) in the system at any point, it had to be present at the very start and fully developed in force and energy.

We can never add more entanglement. This means there has to be an entanglement constant for every participant in the system. This must hold true for the mass of the system, a quantity of mass must be conserved over time [7].

This means there has to be a mass constant and it must be invariant. It must be based on entanglement and it must represent the amount of energy not available to do work in the system or the stored energy in mass. This is also known as Entropy.

\section{Entangled Entropy Function}

$$
s=\frac{1}{\left(1-\frac{1}{e}\right)}
$$

Equation (7)

$e=$ Entangled Energy $=121$. The photon energy of a harmonic series microwave. $S=1.0083333333333333333333333333333$ Entangled Entropy constant.

This simple function labeled as Equation (7) has answered one of the longest lasting and most fundamental questions of science. 
Thus, it is found in a very incredible mathematical triumph that the 121 universal energy is indeed entangled. Take notice of the inverse of 121 and the inverse of the entropy constant 1.008 .

An amazing result of entangled math redefines Mathematics.

Notice in Figure 5 the two inverses are exactly mirrored to infinity.

This quality turns everything we thought about mathematics on its head. We can actually see the numbers fluctuate between the amount of available energy 121 and the entropy of 1.008 .

It is actually showing how objects can have mass and energy at the same time. The 1.008 entropy is the amount of energy that is not available to do work it is otherwise being used and is locked into Mass.

The 1.008 is our invariant mass constant. In mathematics and theoretical physics, an invariant is a property of a system which remains unchanged under some transformation [8].

The invariant mass, rest mass, intrinsic mass, proper mass, or in the case of bound systems simply mass, is the portion of the total mass of an object or system objects that is independent of the overall motion of the system. More precisely, it is a characteristic of the system's total energy and momentum that is the same in all frames of reference. If a center-of-momentum frame exists for the system, then the invariant mass of a system is equal to its total mass in that "rest frame". In other reference frames, where the system's momentum is nonzero, the total mass (a.k.a. relativistic mass) of the system is greater than the invariant mass, but the invariant mass remains unchanged [8].

All thermodynamic systems generate waste heat. This waste results in an increase in entropy, which for a closed system is a quantitative measure of the amount of thermal energy not available to do work [9].

It is found that the answer is eerily similar to the measured energy density of a cubic centimeter of empty space and it is called the cosmological constant and has been repeatedly measured to be at $1 \times 10120$. Per centimeter cubed. $1.008333333333333333 \times 120=121$ the entangled energy constant.

For the first time, we find that this enormous amount of energy is accurate and is available to do work in our universe. This type of oscillation is technically known as complementary, as two opposites have found a way to exist inside the same pole. This shows the entanglement of energy and matter. They display mirrored characteristics like Spin and charge even when separated by time or space. This says a change in one particle is instantly mirrored in the other this makes them complementary.

It should also be considered that the entropy constant of 1.008 is almost an exact match for the mass of the hydrogen atom the smallest atom in the universe.

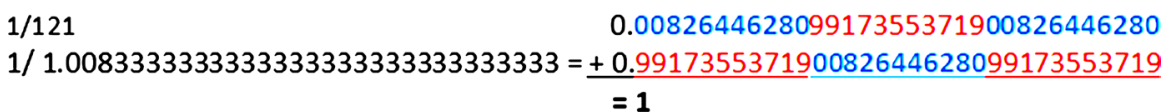

Figure 5. The result is substantiated by the two inverses added $=1$. 
The most basic building block of the universe is the hydrogen atom. $99.9 \%$ of everything in our universe is hydrogen atoms. Thus, the finding that this is the exact amount of mass that is locked and unable to be used, is exactly what it should be, the mass of a hydrogen atom.

One needs to remember that this entire process was begun with the CMB. First we used the $1 \mathrm{~mm}$ diameter of the microwave to make the pattern. Using the photon energy of this same microwave to find the entangled energy. The keyword in $\mathrm{CMB}$ cosmological microwave background is the word microwave. The $\mathrm{CMB}$ is made from a microwave.

Finally, we have seen that we can calculate entanglement. It is just as simple to find that coherence exists in the start of the system as well.

\section{How Is Quantum Coherence Added into the System?}

As demonstrated it is simple to add entanglement and that works for a two-dimensional system. However, it is coherence that takes the entangled system and moves it from two-dimensional to three-dimensional in what I call multi-dimensional wave pattern entangled math.

Don't let the big-name scare you, it actually makes all the math of the universe very simple.

We know that the system is coherent. Thus, coherence had to be there at the start of the system. All we have to do is discover it.

We have only 10 digits at the start of the system. 0123456789 and only nine waves because 0 is not a wave.

We then look at our velocity constant and see that it is 10 digits $9,000,000,000$ $=c^{2}$.

It is shown that the energy constant is $1,210,000,000$ is 10 digits. We find a pattern of 10 digits this looks like coherence.

So when using multi-dimensional entangled wave pattern math we will always reduce the action or the event it can be temperature, velocity, energy, force down to 10 digits. This will create coherence in the system.

Above we used 2.7272727272727272727272727272727 to calculate the entangled exact thermal temperature and it gave us exact entangled energy of 0.134444444444444444444444444444445 combining these two constants to get the entangles universal energy.0.1344444444444444444444444444444445* $C^{2}=$ $12,100,000,000$.

This time we are going to use the same function but instead of starting with 2.7272727272727272727272727272727

We are going to start with 2.727272727

10 digits

2.727272727

You will recognize the temperature constant of the CMB at the start 1,344,444 you don't recognize the other two constants because you have never calculated them, but they are constants. 
Figure 6 is the most amazing part in the history of mathematics.

Now again I will combine this new 3-dimensional constant with our entangled velocity of light.

$$
\begin{aligned}
& 0.1344444444713333333373666666672 \times C^{2} \\
& =12,100,000,002.420000000363000000048
\end{aligned}
$$

The answer gives us the universal entangled energy but in a perfect harmonic series dispersion pattern.

$121 / 242=1 / 2,242 / 363=2 / 3,363 / 486=3 / 4$ you see it is still a perfect harmonic series $1 / 2,2 / 3,3 / 4,4 / 5,5 / 6,6 / 7 \ldots$ to infinity.

Now we ask does this dispersion pattern of the energy match our wave pattern of the hydrogen atom.

$12100000002.420000000363000000048 \times c^{2}$

$=1,089,000,000,217,800,000,032.67000000435600000054450000006534000000762300000087120000009801$

1089-9801, 2178-8712, 3267-7623, 4356-6534 until we reach the center of 5445 this center exactly mirrors itself as 54-45.

Do you remember this wave dispersion pattern from above? It is the hydrogen atom spectrum as calculated above. This demonstrates that our universal energy is indeed in the continuum of the wave and all our in perfect harmonic entangled coherent symmetry.

We added coherence to the system and in so doing we calculated the exact entangled energy dispersion pattern to infinity.

This same exact system will work for any member of the continuum.

Example. Using the 10 digit coherence rule.

Inverting $c^{2}=1.1111111111111111111111111111111 \mathrm{e}-11$

We want coherence so we will use only 10 digits.

$0.111111111 \times c^{2}=0.012345678987654321$

Inverted $=1 / 12345678987654321=81.000000162000000243000000324$

The Hydrogen spectrum. In perfect harmonic series.

We see that our universal velocity is in this same exact pattern of the hydrogen atom as well and is a perfect fit into the continuum.

We know that a microwave creates the wave pattern of 0123456789 we know the $\mathrm{CMB}$ is a microwave thus the wave pattern of a microwave when square rooted $=0.111111111$

This most basic wave 0.111111111 squared $=012345678987654321=$ The entangled CMB wave that makes the hydrogen atom.

This entangled system allows for further simple calculation of entangled energy.

Now we add spin into the continuum.

$$
1 /\left(\frac{\frac{2.727272727}{1}}{2.727272727}\right)=0.1344444444713333333373666666672
$$

Figure 6. Notice the difference in the answer we now have three complete constants in the answer. 


\section{Spin}

We know that spin exists. As a matter of fact, pretty much everything is spinning. We know that all particles spin. We know that for every action there is an equal and opposite reaction. Thus, if a wave is spinning in one direction the center or inverse of the wave must be spinning in the opposite direction. This sounds a bit impossible. However, it is called reactive centripetal $=(0.6666666666)$ and centrifugal forces $=(0.166666666)$. To date, this force is largely ignored but as WPEM will demonstrate not only is it the main force of the universe it is entangled and it fits perfectly into the same wave system of 0123456789 and never breaks the pattern and create the gravitational constant of 9801 .

$$
\begin{gathered}
1 / 0.111111111=9 \\
0.111111111 \times c^{2}=12345678987654321
\end{gathered}
$$

Inverted $1 / 12345678987654321=$ The Entangled Hydrogen shells. $8.1000000162000000243000000324 e-17$

The Entangled Harmonic Hydrogen Spectrum. Applying the Trinity system we can find the two, pole constants and find the spin Constant.

$0.6666666666 / 1 / 0.66666666666=0.44444444444$

$0.166666666 / 1 / 0.166666666=0.0277777777$

We can now combine these two entangled constants.

$0.02777777777 \times 44444444444=0.012345679$ the same hydrogen atom

\section{Conclusions}

It is found that all elements needed to form the entire universe were present in the tiny primordial wave that created the universe, and they were all present in the simple pattern of 0123456789 . They were all present in the simple pattern of 0123456789. This is why we find these qualities in our math. They were there at the very start. By reducing the pattern down the very start we can easily find these phenomena in the pattern, continue the work of our great scientists and find the universal field theory of wave pattern entangled math.

With the inclusion of entanglement, the universe unfolds perfectly, and we finally have an all encompassing unified theory.

This discovery suggests that relativity is not the most fundamental aspect of the universe and the speed of light cannot be the limiting factor that we think it is. Allowing us to go deeper than the laws of Causality and breaking entanglement down to its most fundamental level, we find that Complementarity is the only way for physics to advance; it becomes the fundamental basis for reality. And that relativity is just one of many participants in the continuum created from complementary interaction.

\section{Declaration of Interest Statement}

The author declares that the research was conducted in the absence of any commercial or financial relationships that could be construed as a potential con- 
flict of interest.

\section{References}

[1] Physics Course Book, Experiment 21, Spectrum of Hydrogen and Rydberg Constant. http://personal.tcu.edu/ystrzhemechn/Classes/2010/Summer/Labs/lab21.pdf

[2] Experts, D. (2019) Guide to English \& Logical Reasoning for BITSAT with Past 6 Year Solved Papers. 8th Edition, Disha Publications.

[3] Quantum. The New Quantum Mechanicos of Heisenberg, Schrödinger, and Dirac. https://science.jrank.org/pages/10933/Quantum-New-Quantum-Mechanics-Heisen berg-Schrdinger-Dirac.html

[4] Electromagnetic Spectrum. https://web.pa.msu.edu/courses/2000fall/phy232/lectures/emwaves/spectrum.html

[5] Deepak Chopra, M. and Kafatos, M.C. (2017) You Are the Universe, Discovering Your Cosmic Self and Why It Matters. Harmony Books, New York, 239.

[6] Curium, P.J. (2017) Information at the Center of Creation. In: Traditions, I., et al., Eds., The Cosmic Hologram, Inner Traditions, Rochester, VT, 18.

[7] Revision History Statistics, Bibliographic Details for "Conservation of Mass" (February 2020).

https://en.wikipedia.org/w/index.php?title=Conservation_of_mass\&oldid=9387664 19

[8] Wikipedia, The Free Encyclopedia (2019) Invariant (Physics).

[9] Wikipedia, The Free Encyclopedia (2020) Conservation of Energy. 